\title{
The importance of sagittal balance in adult scoliosis surgery
}

\author{
Jason Pui Yin Cheung \\ Department of Orthopaedics and Traumatology, The University of Hong Kong, Pokfulam, Hong Kong, China \\ Correspondence to: Jason Pui Yin Cheung. Department of Orthopaedics \& Traumatology, The University of Hong Kong, 5th Floor, Professorial Block, \\ 102 Pokfulam Road, Pokfulam, Hong Kong, China. Email: cheungjp@hku.hk.
}

\begin{abstract}
Adult spinal deformity is an important health issue worldwide with our aging population. Understanding ideal sagittal alignment parameters is crucial for planning reconstructive surgery. Despite its variability, sagittal spinopelvic parameters are well recognized as the most crucial factor in predicting postoperative outcomes and risks of revision surgery. Thus, understanding the fundamental concepts of spinopelvic harmony is of utmost importance because they provide useful recommendations for what should be achieved during surgery. The main pathology in degenerative spine disease is the loss of lumbar lordosis (LL), which contributes to lower back pain. The loss of LL may occur as a result of natural history with spinal degeneration or by previous lumbar spine fusion. With adult spinal deformity, understanding the compensatory mechanisms available to patients is important for determining the timing of surgery. The main compensatory mechanisms patients adopt to maintain an upright posture include decreased sacral slope (SS), increased pelvic tilt (PT), decreased thoracic kyphosis (TK). Failure of these compensatory mechanisms leads to recruitment of the lower limbs with flexed hips and knees. At this stage, the patient is decompensated and result in positive sagittal alignment. This sagittal imbalance can be easily measured by the sagittal vertical axis (SVA) and is associated with worse patient-perceived outcome scores. These sagittal parameters also indicate whether surgical reconstruction is required and provides the necessary alignment goals. Depending on the value of pelvic incidence (PI), there are different LL goals. High PI has increased capacity for pelvic retroversion but requires greater lordosis correction. Proper restoration of the LL according to the PI will reduce pelvic retroversion reflected by reduced PT. Without adherence to these surgical goals, complications such as proximal junctional kyphosis (PJK) may occur. It is imperative to restore normal spinopelvic balance to maximize functional outcomes, reduce pain, and avoid complications.
\end{abstract}

Keywords: Sagittal balance; adult spinal deformity; compensatory mechanism; spinopelvic harmony; lumbar lordosis (LL)

Submitted Aug 30, 2019. Accepted for publication Sep 29, 2019.

doi: 10.21037/atm.2019.10.19

View this article at: http://dx.doi.org/10.21037/atm.2019.10.19

\section{Introduction}

Degenerative spinal disorders are now the forefront of most spine practices due to improved medicine and expected high quality of life of our elderly population. The incidence is up to $32 \%$ in adults and $60 \%$ in the elderly (1-3). Most degenerative spinal diseases present with back pain which is now the most common presenting symptom in our clinics and is associated with tremendous financial and social burden on patients and healthcare $(4,5)$. It was identified as the leading global cause of disability in most countries in 2015 and a large percentage can be contributed to deformity (4). As such, the impact of this disease is recognized globally.

Spinal degeneration can manifest as disc disease, facet arthrosis, spinal stenosis, spondylolisthesis, and degenerative scoliosis (6). These conditions usually co-exist and are often triggered by disc disease. This is a degenerative cascade as described by Kirkaldy-Willis. Patients with axial back pain often present with intervertebral degeneration, and significant loss in disc height leads to single or multi-segmental facet joint degeneration due to 
overloading. Eventually, segmental instability occurs with spondylolisthesis. These features may occur at multiple sites leading to adult spinal deformities with scoliosis, flatback, or lumbar kyphosis. In addition to the deformity, patients may have co-existing spinal stenosis which may also give rise to back pain (7).

Due to a growing number of patients with back pain and deformities, there is an increased interest in improving our knowledge of its pathogenesis, alignment objectives, and optimal corrective surgeries to achieve better outcomes. Hence in the past decade, much effort has been made to determine ideal alignment parameters (8-11), fusion level selection, corrective techniques, and instrumentation strategies (12-17).

\section{Spinopelvic harmony}

In adult spinal deformity, sagittal balance is of utmost importance. Human bipedalism is a stable and ergonomic posture. In this posture, the pelvis is a key structure for maintaining stability and directly influences the spinal alignment. The importance of studying the pelvic morphology when describing the sagittal balance cannot be emphasized enough. This is mostly contributed by work from Dubousset and Duval-Beaupère (18). The pelvis is an important segment that intercalates the trunk and lower limbs through the hip joint. It is referred to as the pelvic vertebra by Dubousset due to its close relationship to the spine. The lumbar spine is closely associated with the pelvic position. As humans who adopt a bipedal stance, the lumbar spine in lordosis helps to maintain an upright posture and support the weight. Loading of the spine onto the pelvis is transferred to the lower limbs through the hips. The sacral plateau supports the entire spine and is the point of transfer of loads into the pelvis.

\section{What is positive sagittal balance?}

A forward stooping posture due to the events of failure of compensation is referred to as positive sagittal balance. Patients in this feature often develop increased back pain and worse patient-perceived health-related quality of life scores (19). This positive sagittal balance is a sagittal imbalance which relates to the disruption of the "Cone of Economy" as coined by Jean Dubousset. The cone is projected from the feet up, and so the trunk is only within a narrow range. This concept relates to the part of the cone where the body can remain balanced without external support and using minimal effort. A much greater muscular effort is required to maintain an upright posture when this cone is exceeded, hence leading to more pain and disability. The sagittal vertical axis (SVA) is a commonly used method to quantify sagittal balance and has been shown to result in greater disability (20).

Often patients with adult spinal deformities also have spinal stenosis as a result of foraminal narrowing in scoliosis, and with disc degeneration and spondylolisthesis. It is also important to recognize the inability of patients to stand upright with nerve root compression and associated radiculopathy. In cases with spinal stenosis, patients also adopt a forward standing posture to relieve nerve compression symptoms with reduction of epidural pressure through venous decompression (21). Patients with predominant spinal stenosis symptoms (22) require careful planning to avoid fusing patients without fixed sagittal imbalance.

\section{Radiological parameters for sagittal balance}

Prognostication and surgical planning for adult spinal deformities require a thorough analysis of standing radiographs for sagittal spinopelvic parameters (Figure 1). The width of the pelvis is quantified by the pelvic incidence (PI). The PI is specific to each individual and is anatomically fixed after adolescence. The mean value has been reported as $55 \pm 10$ degrees (23). However, the PI is unique to each individual and should be treated as such. The pelvis rotates around the femoral head and is quantified by the pelvic tilt (PT). In retroversion or backward rotation of the pelvis, the PT increases. With anteversion or forward rotation of the pelvis, the PT decreases. The last pelvic measure is the sacral slope (SS) which indicates the S1 endplate position. The PI determines the relative position of the sacral endplate with the femoral heads. Ideally, the PT should be less than $50 \%$ of the PI and SS should exceed $50 \%$ of PI (24). This way, there is greater potential for compensatory mechanisms with pelvic retroversion. Geometrically, these three measurements are linked with the equation: $\mathrm{PI}=\mathrm{PT}+\mathrm{SS}$.

There are no "normal" pelvic parameters, but a low value of PI is commonly referred to as under 35 degrees while a high value is around 85 degrees (25). There are compensatory implications for low or high PI values. Patients with low PI have a pelvis with a short pelvic ring or small anteroposterior diameter. There is an associated vertical pelvis that is narrow horizontally and strong vertically. In this condition, the femoral heads are just below 


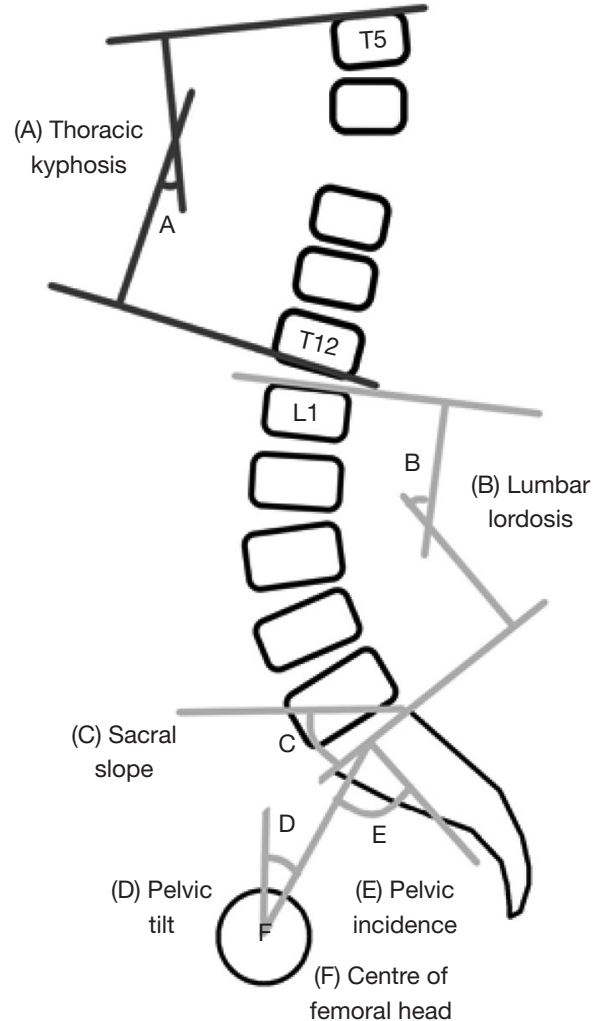

Figure 1 Common spinopelvic parameters: (A) thoracic kyphosis measured from T5-T12; (B) lumbar lordosis measured from L1S1; (C) sacral slope measured by the angle of the S1 endplate with the horizontal; (D) pelvic tilt measured by the angle between a line connecting the center of the femoral heads with the center of the S1 endplate and the vertical; (E) pelvic incidence is the combination of the sacral slope and pelvic tilt and is measured by the angle formed between the (F) centre of femoral head to the midpoint of the S1 endplate and a perpendicular vertical line from the horizontal.

the sacral plate leading to a low SS with the limited ability of the pelvis to tilt. Conversely, a pelvis with a high PI has a large anteroposterior axis with a large horizontal pelvis. The femoral heads are in front of the midpoint of the sacral plate leading to larger SS and higher potential for compensatory retroversion.

Overall spinal balance is often reliant on the relationship between the pelvic parameters and the lumbar lordosis (LL). Schwab et al. (26) suggest that the PI closely matches the LL as measured from L1-S1. However, it is unlikely for the LL to match the PI closely in every patient as variations in spinal alignment do occur. Another concept suggested by Roussouly et al. (27) is the inconsistent location of the

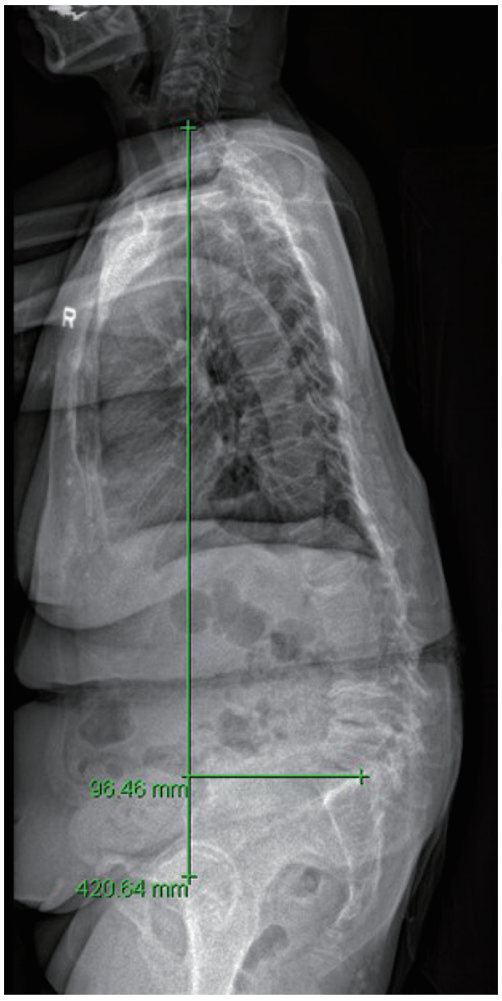

Figure 2 The SVA is measured by the distance between a C7 vertical plumb line with the superior posterior corner of S1. SVA, sagittal vertical axis.

inflection point between LL and thoracic kyphosis (TK). Essentially, the inflection point is not necessarily located at T12-L1 but varies according to the PI. Thus, LL should not always be measured from L1-S1. Rather, the LL should be divided into the upper and lower arches. The lower arch (L4-S1) is clinically more important as up to $70 \%$ of the total LL is located here. It is important to achieve good surgical correction of the lower arch to restore sagittal balance.

The most commonly used measurement to evaluate global spinal balance is the SVA. The SVA is measured by the distance between a C7 vertical plumb line with the superior posterior corner of S1 (Figure 2). The SVA is positive if the line passes $>2 \mathrm{~cm}$ in front of the superior posterior corner of $\mathrm{S} 1$ and is negative if the line passes $>2 \mathrm{~cm}$ behind the superior posterior corner of S1. More than $5 \mathrm{~cm}$ positive SVA is considered abnormal (19). However, this measurement has been criticized for not being representative of the actual center of gravity or balance (28). It is also a measure easily affected by patient posture. The T1 pelvic angle (29) is another tool for assessment of global 


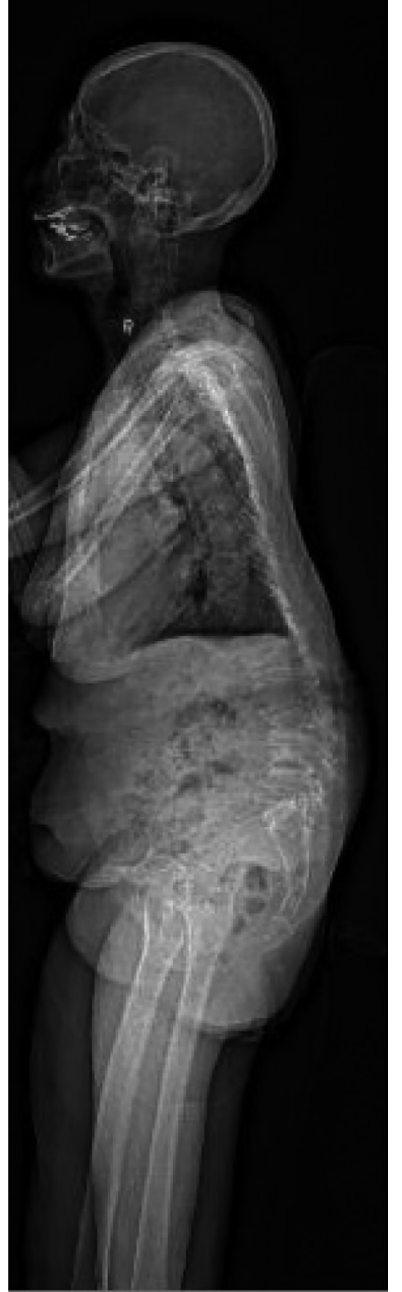

Figure 3 A patient with compensated sagittal balance by utilizing thoracic hypokyphosis and pelvic retroversion (a vertical pelvis with increased pelvic tilt). Slight flexion of the hips and knees are observed.

sagittal balance. It accounts for interaction between SVA and PT, trunk inclination and pelvic retroversion (30). A line is drawn from the center of the T1 vertebral body center to the femoral heads and a line from the femoral heads to the center of the S1 endplate. A target angle of 10-15 degrees for patients aged 40-65 and 15-25 degrees for patients older than 65 can reduce postoperative deterioration in outcomes (30). Other measurements, such as the full balance integrated (FBI) method (31) and gravity line measurement from the external auditory meatus (32) are more comprehensive in assessing balance. The FBI technique is based on a global analysis of the fullbody balance and is a measurement based on the angle of $\mathrm{C} 7$ translation (angle between midpoint of C7 inferior plateau, projected future C7 plumb line and S1 plateau), angle of femur obliquity (inclination of the femoral axis to the vertical) and angle of tilt compensation [based on Roussouly's classification (27)].

There is also growing interest in incorporating the cervical spine assessment with overall balance. Measurements like C2-7 angle and cervical SVA have been studied. However, since the cervical spine is flexible, their clinical implications are uncertain, and further studies are required. Cervical spine movement can be a compensatory mechanism to maintain horizontal gaze (33).

\section{Compensation for sagittal imbalance}

Compensatory mechanisms relate to the severity of the imbalance. It can be balanced, balance with compensatory mechanisms and imbalanced. If the loss of LL is compensated to maintain overall sagittal balance, then it is unlikely for the global alignment to be a cause of pain. When patients lose their normal LL, patients require several compensatory maneuvers (Figure 3) such as hypokyphosis of the thoracic spine, and pelvic retroversion with reduced SS and increased PT to maintain an upright posture (34). If these fail, the lower limbs are recruited for compensation by placing the hips and knees in flexion. Muscular contraction is important to restore balance. Adequate thoracic back musculature is necessary to achieve thoracic hypokyphosis and this may suggest that the thoracic spine can still compensate for any residual deformities and may not require fusion. In decompensation, there is forward tilt (Figure 4) despite pelvic retroversion, with contractures of hamstrings and abdominal muscles. With progressive deformity and back musculature fatigue, patients will adopt a forward stooping posture. Fatigue in the lower limbs with accelerated degeneration of hips and knees may also occur. If the imbalance is a source of pain, correcting the sagittal profile may improve back pain. This will require achieving a more anteverted pelvis while increasing the SS. The compensatory mechanisms of pelvic retroversion, increased PT and decreased TK may spontaneously correct after successful restoration of LL $(35,36)$. The legs will also be able to straighten.

\section{Classification of adult spinal deformity}

The most commonly used classification for adult spinal deformities is the SRS-Schwab classification (26), which 


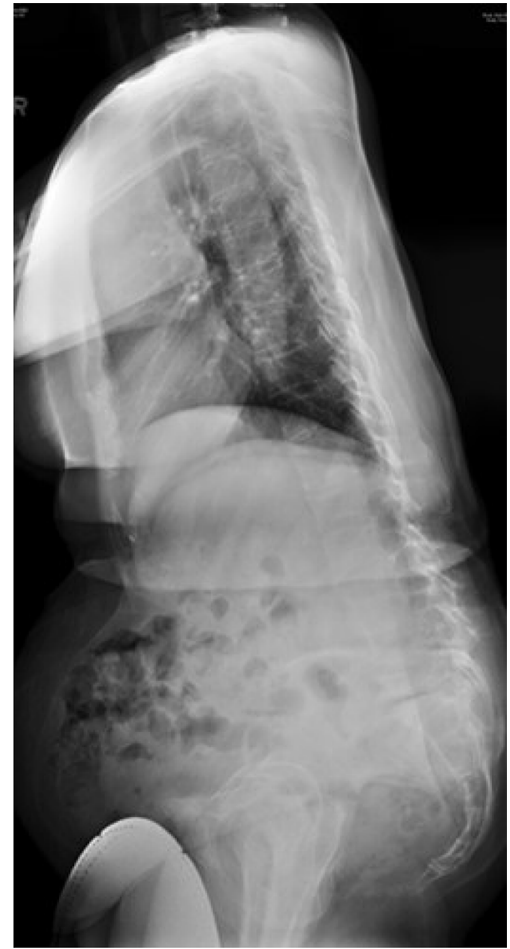

Figure 4 A patient with uncompensated sagittal balance with forward posture despite thoracic hypokyphosis and pelvic retroversion.

is descriptive. It consists of components split between the coronal curve types and sagittal modifiers. There are four components: coronal curve type, PI-LL modifier, PT modifier, and global balance modifier. For the coronal curve type, patients are divided into thoracic only (with a lumbar curve less than 30 degrees), thoracolumbar/lumbar only (with a thoracic curve less than 30 degrees), double curve (thoracic and thoracolumbar/lumbar curves both more than 30 degrees) or no coronal curve (all coronal curves less than 30 degrees). The PI-LL mismatch is graded as 0 (PI-LL less than 10 degrees), + (PI-LL between 10-20 degrees) and ++ (PI-LL more than 20 degrees). The global balance modifier is measured by the C7-S1 SVA. It is graded as 0 (SVA less than $4 \mathrm{~cm}),+($ SVA between 4-9.5 $\mathrm{cm})$ and ++ (SVA more than $9.5 \mathrm{~cm}$ ). Finally, the PT is graded as 0 (PT less than 20 degrees), + (PT between 20-30 degrees) and ++ (PT more than 30 degrees). This is now a validated tool for surgical planning and communication. It has also been shown to correlate well with health-related quality of life measures (37).

The sagittal profile of the spine is not simply described as a kyphotic thoracic spine and lordotic lumbar spine. A more intuitive classification is proposed by Roussouly (27) based on four types of sagittal alignment determined by the location of the inflection point. The LL is divided into an upper arc and lower arc defined by the apex of the lumbar curve towards the position of the inflection point and the SS respectively. The angle between the sacral plate and the inflection point is called the lordosis tilt angle. The four types are named type 1 lordosis, type 2 lordosis, type 3 lordosis, and type 4 lordosis. For type 1 lordosis, the SS is less than 35 degrees with a low PI. The apex is at the L5, and thus the inflection point is low, and the lower arc of lordosis is minimal. There is kyphosis at the thoracolumbar junction. In type 2 lordosis, the SS is less than 35 degrees, but the apex is at L4. The inflection is higher and more anterior, but the lower arc of lordosis is flat. The spine is generally flatback. For type 3 lordosis, the SS is between 35 and 45 degrees. The apex is also at L4, but the lower arc of lordosis is more prominent than the previous types. The spine is well balanced, and the inflection point is at the thoracolumbar junction. Finally, for type 4 lordosis, there is a SS of greater than 45 degrees with a high PI. The apex is at L3 or higher with a prominent lower arc of lordosis. The LL segment includes more proximal segments than other types. This classification has major implications for the standard of corrective surgery as with types 1 and 2, a larger burden of correction on the lower segments is required as compared to type 4 which has more vertebrae to compensate.

\section{Any role for conservative treatment?}

Nonoperative management is usually reserved for patients with mild symptoms arising from stenosis, radicular or back pain, curve magnitude of fewer than 30 degrees, less than $2 \mathrm{~mm}$ of lateral subluxation, and reasonable coronal and sagittal balance (38). Maximizing conservative treatment before operative treatment is necessary with the high risk of surgical complications up to $80 \%$ with a reoperation rate of $50 \%(39,40)$. This is especially important when taking into account the high cost of these surgeries (41).

Several studies (42-46) have compared outcomes of surgical and nonoperative cohorts. However, these studies carry a high risk of selection bias, and none of them provided a standardized conservative treatment protocol, details regarding what therapies and training were given, and explanation of what was considered a failure of conservative treatment (47). Of note, when and 

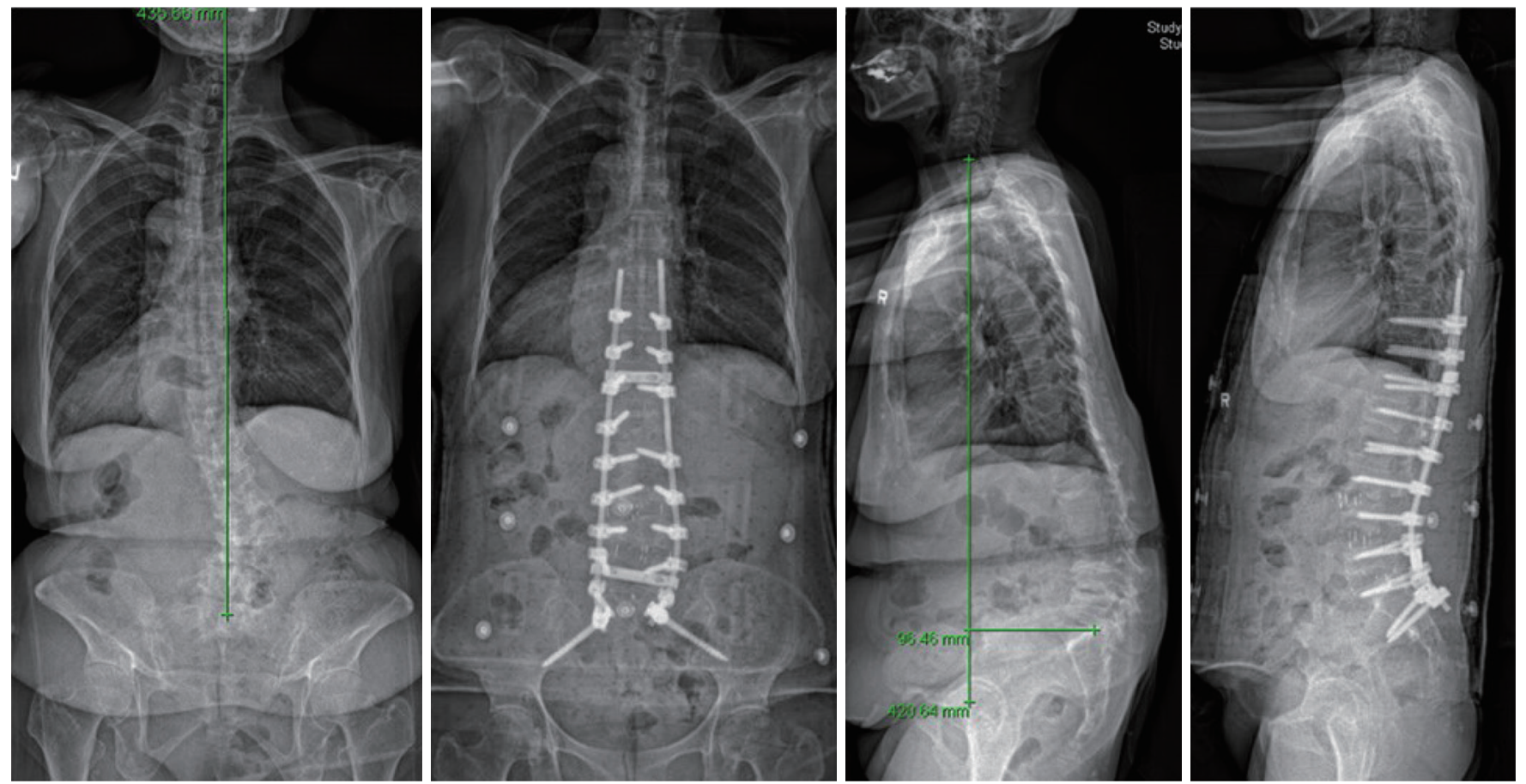

Figure 5 A patient with a preoperative pelvic incidence of 45 degrees, a pelvic tilt of 51.9 degrees, the sacral slope of -7.8 degrees, lumbar kyphosis of 28.7 degrees and sagittal vertical axis over $+10 \mathrm{~cm}$. She underwent L3-S1 oblique interbody fusions and posterior instrumentation from T10 to pelvis. Postoperatively, the lumbar lordosis returned to 47 degrees with a pelvic tilt of 20 degrees. The sagittal vertical axis is now centered over the posterior-superior corner of S1.

what to prescribe for conservative treatment and its effect on outcomes is unknown. Most of this available evidence is also retrospective (48), and lack consensus on what is most efficacious for adult deformity and which patients will benefit most. There are many conservative management options, such as bracing, manipulation, physical therapy, and epidural injections (48). Also, the above studies are based on Caucasian populations, and no study has been performed in the Asia-Pacific population. There are obvious ethnic and cultural variations that come into play for degenerative disorders, and such comparative evidence is necessary $(49,50)$. However, these studies all supported a clear message that patients with adequate compensation may avoid surgical interventions. Back musculature can still be trained to maintain a compensated posture.

\section{Aim of surgical correction}

Corrective surgeries aim to reconstitute the spinopelvic harmony to maintain sagittal balance (Figure 5). The lower lumbar spine, in particular, has a high potential for rebalancing surgery as two-thirds of LL is located between L4-S1 (51). How much correction is needed; however, is not well understood and is often subjected to debate. Schwab recommends an SVA $<40 \mathrm{~mm}$, a PI-LL mismatch within 10 degrees and a PT $<20$ degrees for ideal sagittal alignment (26). However, this has been challenged due to variations with age and sex. In a study of asymptomatic volunteers, older patients especially reaching the 60-80 age group had less amount of LL (52). Based on regression analyses of PT, PI-LL mismatch and SVA, age and commonly used health-related quality of life measures of the Oswestry Disability Index (ODI) and 36-item short-form questionnaire (SF-36), younger patients likely require more rigorous alignment objectives than the elderly $(53,54)$. Elderly patients tolerated greater magnitudes of sagittal malalignment. Patients aged 75 years or above can tolerate a PI-LL mismatch of 8.3 with ODI of 20, while for the same ODI, patients 35 to 44 years of age requires a PI-LL of -2.7 (Table 1) (10).

In additional to age-related variations, there is a limitation of using single static standing radiographs for analyses. These radiographs do not consider the spinal 
Table 1 Age-adjusted targets

\begin{tabular}{lccc}
\hline Age group & PT & PI-LL & SVA \\
\hline$<35$ & 11.0 & -10.5 & -30.5 \\
$35-44$ & 15.4 & -4.6 & -5.5 \\
$45-54$ & 18.8 & 0.5 & 15.1 \\
$55-64$ & 22.0 & 5.8 & 35.8 \\
$65-74$ & 25.1 & 10.5 & 54.5 \\
$\geq 74$ & 28.8 & 17.0 & 79.3 \\
\hline
\end{tabular}

PT, pelvic tilt; PI, pelvic incidence; LL, lumbar lordosis; SVA, sagittal vertical axis.

Table 2 Goals of sagittal realignment surgery

\begin{tabular}{lc}
\hline $\mathrm{PI}$ & Equation \\
\hline$<35$ degrees & $\mathrm{LL}=\mathrm{PI}+10$ \\
$35-50$ degrees & $\mathrm{LL}=\mathrm{PI}$ \\
$50-80$ degrees & $\mathrm{LL}=\mathrm{PI}-10$ \\
\hline
\end{tabular}

$\mathrm{PI}$, pelvic incidence; LL, lumbar lordosis.

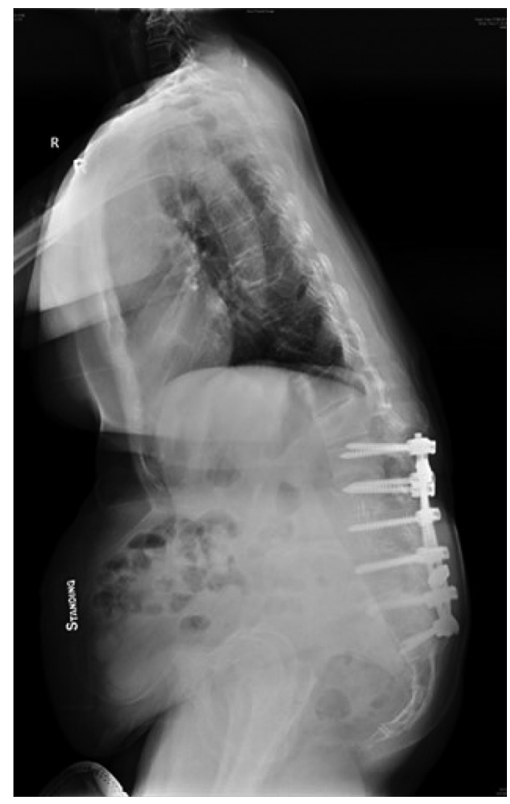

Figure 6 A patient with inadequate correction of the lumbar lordosis in the presence of a high pelvic incidence. There is evidence of proximal junctional kyphosis.

flexibility and functional demands on the spine. Also, most of the postures we adopt in daily life are not standing. One study suggests that in a natural sitting posture, the lumbar spine becomes kyphotic (55). Hence, the LL cannot be a standard measurement due to variations with posture $(25,56)$.

Nevertheless, a commonly used recommendation for sagittal realignment surgery is PI-LL mismatch. Depending on the value of PI, the expected LL may be different (Table 2). A small PI may require a larger LL but a large PI may require a smaller LL for balance. Equations such as $\mathrm{LL}=\mathrm{PI}+10$ for small PI, LL $=$ PI for average PI, and $\mathrm{LL}=\mathrm{PI}-10$ for large PI $\sim 50-80$ degrees have been proposed (57).

Osteotomies are commonly employed to restore sagittal balance. Depending on the amount of correction needed, the type of osteotomies may be planned appropriately to achieve the intended sagittal correction. Posterior column osteotomies involve the removal of posterior ligaments with a complete facetectomy. The amount of sagittal correction is usually 5-10 degrees per level depending on how liberal is the osteotomy and the degree of flexibility in the disc space. These are usually indicated for more flexible spinal deformities with less amount of correction needed. Three column osteotomies like the pedicle subtraction osteotomy (PSO) are reserved for large sagittal malalignments with fixed and angular deformities. A single PSO can usually generate 20-40 degrees of LL change (58). For patients with less LL angle increases ( $<40$ degrees), greater postoperative SVA $(>8 \mathrm{~cm})$, and reduced differences between LL and TK ( $<25$ degrees) after surgery, sagittal decompensation is more common $(59,60)$. Vertebral column osteotomies usually can correct from 40-80 degrees of kyphosis deformity especially at the lumbosacral and thoracolumbar spine. However, its complication rates are notoriously high.

\section{Complications}

Surgery for adult spinal deformity carries a high risk of surgical complications and reoperation $(39,40)$. Of these, adjacent segment degeneration, inadequate sagittal realignment, and proximal junctional kyphosis (PJK) (Figure 6) are commonly observed. Adjacent segment degeneration after fusion may also be influenced by inappropriate sagittal balance after surgery. There is a higher rate of adjacent segment degeneration in patients with an abnormal SVA (61). Lumbar spinal fusions, in general, can lead to problems in the sagittal spinal balance due to reduced SS and LL $(56,62)$. Lumbar fusions that result in an improper postoperative LL or simply unchanged LL, especially from L4-S1, will result in increased LL at the upper unfused segments at L1-4. A more vertical sacrum with reduced SS and increased PT 
results in more post-fusion pain (56).

Flat-back can result from loss of lordosis after fusion. Hence, the spinopelvic parameters should be carefully considered preoperatively with the successful execution of the correction surgery to restore LL and prevent fixed sagittal imbalance. Cho et al. (63). showed that patients with a high PI are at highest risk for sagittal decompensation due to surgical under-correction. Patients with higher PI may need more LL correction for sagittal balancing (64). This may reduce the need for thoracic hypokyphosis at the risk of PJK. (36). $\mathrm{Up}$ to 70-80 degrees of LL restoration may be required to correct sagittal balance. A simple recommendation proposed is $\mathrm{LL}(\mathrm{L} 1-\mathrm{S} 1)=\mathrm{PI}+9$ degrees (65).

Despite osteotomies, often in patients with high PT and malalignment preoperatively fail to restore an acceptable alignment (66). As a result, PJK may occur, especially in long constructs after adult spinal deformity surgery. Hypokyphosis of the thoracic spine because of overcorrection may lead to increasing kyphotic forces at the proximal junction to restore standing balance (67). This may lead to revision surgery and ultimately, worse outcomes. Specifically, for PJK, age-adjusted alignment goals are necessary since older patients do not require as much correction since they are inherently more kyphotic. Lafage et al. (53). have shown that patients with PJK often have smaller postoperative PI-LL mismatches indicating overcorrection during surgery as the main culprit. In addition to alignment changes, patients with osteoporosis, larger TK, paraspinal muscle atrophy and fatty degeneration may all contribute to the risk of PJK (68-70). Hence, PJK is a complex problem that currently has no good prevention. Elderly patients with the osteoporotic bone are more at risk for developing PJK.

It is also important to consider the patient's ability to compensate for any residual deformities beyond the radiological parameters. Hip range of motion and muscle contractures, especially hamstrings, may limit compensation and should be maximized preoperatively to improve postoperative outcomes. This is especially true for extensive long-construct instrumentation as patients lose their ability to adopt compensatory postures. Hence, appropriate surgical realignment should be achieved in the initial surgery, or else revision surgery becomes unavoidable.

\section{Conclusions}

Management of adult spinal deformity requires a proper understanding of the dynamics between the spine and the pelvis, and understanding of sagittal radiological alignment parameters. In the recent decade, there is growing literature regarding various spinopelvic measurements to assess the degree of sagittal imbalance. Regardless of the classification system or parameters used, it is always important to match the LL with the PI. It is important to understand that this relationship is fluid and will change according to patient age and degree of PI. Compensatory mechanisms, including reduced TK, pelvic retroversion with increased PT, and finally hip and joint positioning, indicate when the patient has reached the threshold where interventions may be required. The PI predicts how much compensation is available to patients. Low PI has limited capacity for retroversion as compared to high PI. Alignment goals are also different for low and high PI. Restoring lordosis proportional to the PI value is essential. Those with high PI will require more significant lumbar correction and is thus technically more demanding during surgery. The PI is a fixed anatomical parameter but is reflected by changes in PT and SS. Surgeons can only restore the appropriate LL to influence values of PT and SS. There are many different surgical techniques, but the objective is the same, to achieve spinopelvic harmony. Inability to understand these relationships and inadequate surgical correction to match these goals will result in complications such as PJK.

\section{Acknowledgments}

None.

\section{Footnote}

Conflicts of Interest: The author has no conflicts of interest to declare.

Ethical Statement: The author is accountable for all aspects of the work in ensuring that questions related to the accuracy or integrity of any part of the work are appropriately investigated and resolved.

\section{References}

1. Schwab F, Dubey A, Gamez L, et al. Adult scoliosis: prevalence, SF-36, and nutritional parameters in an elderly volunteer population. Spine (Phila Pa 1976) 2005;30:1082-5.

2. Schwab F, Dubey A, Pagala M, et al. Adult scoliosis: a health assessment analysis by SF-36. Spine (Phila Pa 1976) 2003;28:602-6. 
3. Schwab FJ, Lafage V, Farcy JP, et al. Predicting outcome and complications in the surgical treatment of adult scoliosis. Spine (Phila Pa 1976) 2008;33:2243-7.

4. GBD 2015 Disease and Injury Incidence and Prevalence Collaborators. Global, regional, and national incidence, prevalence, and years lived with disability for 310 diseases and injuries, 1990-2015: a systematic analysis for the Global Burden of Disease Study 2015 Lancet. 2016;388:1545-602.

5. Jackson T, Thomas S, Stabile V, et al. A Systematic Review and Meta-Analysis of the Global Burden of Chronic Pain Without Clear Etiology in Low- and MiddleIncome Countries: Trends in Heterogeneous Data and a Proposal for New Assessment Methods. Anesth Analg 2016;123:739-48.

6. Sasiadek MJ, Bladowska J. Imaging of degenerative spine disease--the state of the art. Adv Clin Exp Med 2012;21:133-42.

7. Cheung JP, Cheung PW, Cheung KM, et al. Decompression without Fusion for Low-Grade Degenerative Spondylolisthesis. Asian Spine J 2016;10:75-84.

8. Ferrero E, Liabaud B, Challier V, et al. Role of pelvic translation and lower-extremity compensation to maintain gravity line position in spinal deformity. J Neurosurg Spine 2016;24:436-46.

9. Glassman SD, Coseo MP, Carreon LY. Sagittal balance is more than just alignment: why PJK remains an unresolved problem. Scoliosis Spinal Disord 2016;11:1.

10. Lafage R, Schwab F, Challier V, et al. Defining SpinoPelvic Alignment Thresholds: Should Operative Goals in Adult Spinal Deformity Surgery Account for Age? Spine (Phila Pa 1976) 2016;41:62-8.

11. Scheer JK, Lafage V, Smith JS, et al. Maintenance of radiographic correction at 2 years following lumbar pedicle subtraction osteotomy is superior with upper thoracic compared with thoracolumbar junction upper instrumented vertebra. Eur Spine J 2015;24 Suppl 1:S121-30.

12. Cho KJ, Suk SI, Park SR, et al. Short fusion versus long fusion for degenerative lumbar scoliosis. Eur Spine J 2008; 17:650-6.

13. Gupta MC, Ferrero E, Mundis G, et al. Pedicle Subtraction Osteotomy in the Revision Versus Primary Adult Spinal Deformity Patient: Is There a Difference in Correction and Complications? Spine (Phila Pa 1976) 2015;40:E1169-75.

14. Isaacs RE, Hyde J, Goodrich JA, et al. A prospective, nonrandomized, multicenter evaluation of extreme lateral interbody fusion for the treatment of adult degenerative scoliosis: perioperative outcomes and complications. Spine (Phila Pa 1976) 2010;35:S322-30.

15. Lafage V, Schwab F, Vira S, et al. Does vertebral level of pedicle subtraction osteotomy correlate with degree of spinopelvic parameter correction? J Neurosurg Spine 2011;14:184-91.

16. Patel R, Khan SN, McMains MC, et al. Technique for Lumbar Pedicle Subtraction Osteotomy for Sagittal Plane Deformity in Revision. Am J Orthop (Belle Mead NJ) 2015;44:261-4.

17. Theologis AA, Mundis GM Jr, Nguyen S, et al. Utility of multilevel lateral interbody fusion of the thoracolumbar coronal curve apex in adult deformity surgery in combination with open posterior instrumentation and L5-S1 interbody fusion: a case-matched evaluation of 32 patients. J Neurosurg Spine 2017;26:208-19.

18. Legaye J, Duval-Beaupère G, Hecquet J, et al. Pelvic incidence: a fundamental pelvic parameter for threedimensional regulation of spinal sagittal curves. Eur Spine J 1998;7:99-103.

19. Glassman SD, Berven S, Bridwell K, et al. Correlation of radiographic parameters and clinical symptoms in adult scoliosis. Spine (Phila Pa 1976) 2005;30:682-8.

20. Glassman SD, Bridwell K, Dimar JR, et al. The impact of positive sagittal balance in adult spinal deformity. Spine (Phila Pa 1976) 2005;30:2024-9.

21. Suzuki H, Endo K, Kobayashi H, et al. Total sagittal spinal alignment in patients with lumbar canal stenosis accompanied by intermittent claudication. Spine (Phila $\mathrm{Pa}$ 1976) 2010;35:E344-6.

22. Cheung PWH, Fong HK, Wong CS, et al. The influence of developmental spinal stenosis on the risk of re-operation on an adjacent segment after decompression-only surgery for lumbar spinal stenosis. Bone Joint J 2019;101-B:154-61.

23. Vialle R, Levassor N, Rillardon L, et al. Radiographic analysis of the sagittal alignment and balance of the spine in asymptomatic subjects. J Bone Joint Surg Am 2005;87:260-7.

24. Mac-Thiong JM, Roussouly P, Berthonnaud E, et al. Sagittal parameters of global spinal balance: normative values from a prospective cohort of seven hundred nine Caucasian asymptomatic adults. Spine (Phila Pa 1976) 2010;35:E1193-8.

25. Vaz G, Roussouly P, Berthonnaud E, et al. Sagittal morphology and equilibrium of pelvis and spine. Eur Spine 
J 2002;11:80-7.

26. Schwab F, Ungar B, Blondel B, et al. Scoliosis Research Society-Schwab adult spinal deformity classification: a validation study. Spine (Phila Pa 1976) 2012;37:1077-82.

27. Roussouly P, Gollogly S, Berthonnaud E, et al. Classification of the normal variation in the sagittal alignment of the human lumbar spine and pelvis in the standing position. Spine (Phila Pa 1976) 2005;30:346-53.

28. Roussouly P, Gollogly S, Noseda O, et al. The vertical projection of the sum of the ground reactive forces of a standing patient is not the same as the $\mathrm{C} 7$ plumb line: a radiographic study of the sagittal alignment of 153 asymptomatic volunteers. Spine (Phila $\mathrm{Pa} 1976$ ) 2006;31:E320-5.

29. Qiao J, Zhu F, Xu L, et al. T1 pelvic angle: a new predictor for postoperative sagittal balance and clinical outcomes in adult scoliosis. Spine (Phila Pa 1976) 2014;39:2103-7.

30. Ryan DJ, Protopsaltis TS, Ames CP, et al. T1 pelvic angle (TPA) effectively evaluates sagittal deformity and assesses radiographical surgical outcomes longitudinally. Spine (Phila Pa 1976) 2014;39:1203-10.

31. Le Huec JC, Leijssen P, Duarte M, et al. Thoracolumbar imbalance analysis for osteotomy planification using a new method: FBI technique. Eur Spine J 2011;20 Suppl 5:669-80.

32. Lafage V, Schwab F, Skalli $W$, et al. Standing balance and sagittal plane spinal deformity: analysis of spinopelvic and gravity line parameters. Spine (Phila $\mathrm{Pa} 1976$ ) 2008;33:1572-8.

33. Blondel B, Schwab F, Bess S, et al. Posterior global malalignment after osteotomy for sagittal plane deformity: it happens and here is why. Spine (Phila Pa 1976) 2013;38:E394-401.

34. Rajnics P, Templier A, Skalli W, et al. The association of sagittal spinal and pelvic parameters in asymptomatic persons and patients with isthmic spondylolisthesis. J Spinal Disord Tech 2002;15:24-30.

35. Godde S, Fritsch E, Dienst M, et al. Influence of cage geometry on sagittal alignment in instrumented posterior lumbar interbody fusion. Spine (Phila $\mathrm{Pa}$ 1976) 2003;28:1693-9.

36. Jang JS, Lee SH, Min JH, et al. Influence of lumbar lordosis restoration on thoracic curve and sagittal position in lumbar degenerative kyphosis patients. Spine (Phila $\mathrm{Pa}$ 1976) 2009;34:280-4.

37. Smith JS, Klineberg E, Schwab F, et al. Change in classification grade by the SRS-Schwab Adult Spinal Deformity Classification predicts impact on health-related quality of life measures: prospective analysis of operative and nonoperative treatment. Spine (Phila $\mathrm{Pa} 1976$ ) 2013;38:1663-71.

38. Silva FE, Lenke LG. Adult degenerative scoliosis: evaluation and management. Neurosurg Focus 2010;28:E1.

39. Carreon LY, Puno RM, Dimar JR 2nd, et al. Perioperative complications of posterior lumbar decompression and arthrodesis in older adults. J Bone Joint Surg Am 2003;85A:2089-92.

40. Edwards CC, 2nd, Bridwell KH, Patel A, et al. Long adult deformity fusions to L5 and the sacrum. A matched cohort analysis. Spine (Phila Pa 1976) 2004;29:1996-2005.

41. McCarthy I, Hostin R, O'Brien M, et al. Health economic analysis of adult deformity surgery. Neurosurg Clin N Am 2013;24:293-304.

42. Acaroglu E, Yavuz AC, Guler UO, et al. A decision analysis to identify the ideal treatment for adult spinal deformity: is surgery better than non-surgical treatment in improving health-related quality of life and decreasing the disease burden? Eur Spine J 2016;25:2390-400.

43. Kluba T, Dikmenli G, Dietz K, et al. Comparison of surgical and conservative treatment for degenerative lumbar scoliosis. Arch Orthop Trauma Surg 2009;129:1-5.

44. Liu S, Diebo BG, Henry JK, et al. The benefit of nonoperative treatment for adult spinal deformity: identifying predictors for reaching a minimal clinically important difference. Spine J 2016;16:210-8.

45. Neuman BJ, Baldus C, Zebala LP, et al. Patient Factors That Influence Decision Making: Randomization Versus Observational Nonoperative Versus Observational Operative Treatment for Adult Symptomatic Lumbar Scoliosis. Spine (Phila Pa 1976) 2016;41:E349-58.

46. Slobodyanyuk K, Poorman CE, Smith JS, et al. Clinical improvement through nonoperative treatment of adult spinal deformity: who is likely to benefit? Neurosurg Focus 2014;36:E2.

47. Teles AR, Mattei TA, Righesso O, et al. Effectiveness of Operative and Nonoperative Care for Adult Spinal Deformity: Systematic Review of the Literature. Global Spine J 2017;7:170-8.

48. Everett CR, Patel RK. A systematic literature review of nonsurgical treatment in adult scoliosis. Spine (Phila $\mathrm{Pa}$ 1976) 2007;32:S130-4.

49. Williams R, Cheung JP, Goss B, et al. An International Multicenter Study Assessing the Role of Ethnicity on Variation of Lumbar Facet Joint Orientation and the Occurrence of Degenerative Spondylolisthesis in Asia Pacific: A Study from the AOSpine Asia Pacific Research 
Collaboration Consortium. Global Spine J 2016;6:35-45.

50. Samartzis D, Cheung JP, Rajasekaran S, et al. Is lumbar facet joint tropism developmental or secondary to degeneration? An international, large-scale multicenter study by the AOSpine Asia Pacific Research Collaboration Consortium. Scoliosis Spinal Disord 2016;11:9.

51. Harrison DE, Cailliet R, Harrison DD, et al. Changes in sagittal lumbar configuration with a new method of extension traction: nonrandomized clinical controlled trial. Arch Phys Med Rehabil 2002;83:1585-91.

52. Yeh KT, Lee RP, Chen IH, et al. Are There Age- and Sex-related Differences in Spinal Sagittal Alignment and Balance Among Taiwanese Asymptomatic Adults? Clin Orthop Relat Res 2018;476:1010-7.

53. Lafage R, Schwab F, Glassman S, et al. Age-Adjusted Alignment Goals Have the Potential to Reduce PJK. Spine (Phila Pa 1976) 2017;42:1275-82.

54. Passias PG, Jalai CM, Diebo BG, et al. Full-Body Radiographic Analysis of Postoperative Deviations From Age-Adjusted Alignment Goals in Adult Spinal Deformity Correction and Related Compensatory Recruitment. Int J Spine Surg 2019;13:205-14.

55. Hey HW, Wong CG, Lau ET, et al. Differences in erect sitting and natural sitting spinal alignment-insights into a new paradigm and implications in deformity correction. Spine J 2017;17:183-9.

56. Lazennec JY, Ramare S, Arafati N, et al. Sagittal alignment in lumbosacral fusion: relations between radiological parameters and pain. Eur Spine J 2000;9:47-55.

57. Lee CS, Chung SS, Park SJ, et al. Simple prediction method of lumbar lordosis for planning of lumbar corrective surgery: radiological analysis in a Korean population. Eur Spine J 2014;23:192-7.

58. Kim YJ, Bridwell KH, Lenke LG, et al. Results of lumbar pedicle subtraction osteotomies for fixed sagittal imbalance: a minimum 5-year follow-up study. Spine (Phila Pa 1976) 2007;32:2189-97.

59. Lee SH, Kim KT, Suk KS, et al. Sagittal decompensation after corrective osteotomy for lumbar degenerative kyphosis: classification and risk factors. Spine (Phila $\mathrm{Pa}$ 1976) 2011;36:E538-44.

60. Rose PS, Bridwell KH, Lenke LG, et al. Role of pelvic incidence, thoracic kyphosis, and patient factors on sagittal plane correction following pedicle subtraction osteotomy. Spine (Phila Pa 1976) 2009;34:785-91.
61. Kumar MN, Baklanov A, Chopin D. Correlation between sagittal plane changes and adjacent segment degeneration following lumbar spine fusion. Eur Spine J 2001;10:314-9.

62. Kawakami M, Tamaki T, Ando M, et al. Lumbar sagittal balance influences the clinical outcome after decompression and posterolateral spinal fusion for degenerative lumbar spondylolisthesis. Spine (Phila Pa 1976) 2002;27:59-64.

63. Cho KJ, Suk SI, Park SR, et al. Risk factors of sagittal decompensation after long posterior instrumentation and fusion for degenerative lumbar scoliosis. Spine (Phila Pa 1976) 2010;35:1595-601.

64. Le Huec JC, Charosky S, Barrey C, et al. Sagittal imbalance cascade for simple degenerative spine and consequences: algorithm of decision for appropriate treatment. Eur Spine J 2011;20 Suppl 5:699-703.

65. Schwab F, Lafage V, Patel A, et al. Sagittal plane considerations and the pelvis in the adult patient. Spine (Phila Pa 1976) 2009;34:1828-33.

66. Schwab FJ, Patel A, Shaffrey CI, et al. Sagittal realignment failures following pedicle subtraction osteotomy surgery: are we doing enough?: Clinical article. J Neurosurg Spine 2012;16:539-46.

67. Kim HJ, Bridwell KH, Lenke LG, et al. Proximal junctional kyphosis results in inferior SRS pain subscores in adult deformity patients. Spine (Phila $\mathrm{Pa} 1976$ ) 2013;38:896-901.

68. Hyun SJ, Kim YJ, Rhim SC. Patients with proximal junctional kyphosis after stopping at thoracolumbar junction have lower muscularity, fatty degeneration at the thoracolumbar area. Spine J 2016;16:1095-101.

69. Kim HJ, Boachie-Adjei O, Shaffrey CI, et al. Upper thoracic versus lower thoracic upper instrumented vertebrae endpoints have similar outcomes and complications in adult scoliosis. Spine (Phila $\mathrm{Pa} 1976$ ) 2014;39:E795-9.

70. Kim YJ, Bridwell KH, Lenke LG, et al. Sagittal thoracic decompensation following long adult lumbar spinal instrumentation and fusion to L5 or S1: causes, prevalence, and risk factor analysis. Spine (Phila Pa 1976) 2006;31:2359-66.

Cite this article as: Cheung JPY. The importance of sagittal balance in adult scoliosis surgery. Ann Transl Med 2020;8(2):35. doi: 10.21037/atm.2019.10.19 